\title{
Incremental Flattening for Nested Data Parallelism
}

\author{
Troels Henriksen \\ University of Copenhagen \\ athas@sigkill.dk \\ Martin Elsman \\ University of Copenhagen \\ mael@di.ku.dk
}

\author{
Frederik Thorøe \\ University of Copenhagen \\ vbj532@alumni.ku.dk \\ Cosmin Oancea \\ University of Copenhagen \\ cosmin.oancea@diku.dk
}

\begin{abstract}
This artifact contains scripts and programs for reproducing the central experimental figures of the paper (Figures 2, 7 and 8), which show how Futhark programs perform compared to hand-written GPU programs, using different compilation techniques.

This artifact requires access to a computer with a modern GPU (a laptop will likely not cut it) with OpenCL and optionally CUDA already set up and working. Apart from that, the software requirements are fairly standard. A Dockerfile is provided that will work on Linux systems with NVIDIA GPUs, assuming NVIDIAs docker runtime has been installed. This is typically not problematic.

The artifact is structured around a Makefile with targets for the figures and supporting data. The enclosed README.md contains further details and hints about execution and troubleshooting. The artifact is also publicly available at https: //github.com/diku-dk/futhark-ppopp19.
\end{abstract}

CCS Concepts - Computing methodologies $\rightarrow$ Parallel programming languages; - Software and its engineering $\rightarrow$ Source code generation; Software performance;

Keywords functional language, parallel, compilers, GPGPU.

\section{Artifact Appendix}

\subsection{Artifact check-list (meta-information)}

- Program: Rodinia and FinPar; automatically downloaded and patched by scripts.

- Compilation: Requires gcc (or compatible) able to compile OpenCL programs; optionally also nvcc.

Permission to make digital or hard copies of all or part of this work for personal or classroom use is granted without fee provided that copies are not made or distributed for profit or commercial advantage and that copies bear this notice and the full citation on the first page. Copyrights for components of this work owned by others than the author(s) must be honored. Abstracting with credit is permitted. To copy otherwise, or republish, to post on servers or to redistribute to lists, requires prior specific permission and/or a fee. Request permissions from permissions@acm.org. PPoPP '19, February 16-20, 2019, Washington, DC, USA

(c) 2019 Copyright held by the owner/author(s). Publication rights licensed to the Association for Computing Machinery. ACM ISBN 978-1-4503-6225-2/19/02 ..\$15.00 https://doi.org/10.1145/3293883.3295707
- Run-time environment: Requires Linux (some parts may work on macOS). Docker image may require root access (unclear; we are not Docker experts). A UTF-8 enabled locale is required (e.g. set environment variable LC_ALL=en_US.UTF-8).

- Hardware: We require a recent-ish NVIDIA or AMD GPU, ideally with $4 \mathrm{GiB}$ or more memory (README describes how to run the less memory-intensive parts on smaller GPUs). Some of our dependencies may require an x86 CPU.

- Execution: Full execution takes from one to three hours depending on system speed.

- Metrics: We measure wall-clock runtimes, and the enclosed plotting scripts measure speedup compared to a computed baseline.

- Output: We produce speedup graphs in PDF format, and raw measurement data in a simple JSON format.

- Experiments: A Dockerfile is provided for use on NVIDIA systems, which has also been made available on Docker Hub for ease of use. Ideally, running the experiments and generating the graphs is just make.

- How much disk space required (approximately)?: The artifact itself is small, but running it will take more than $10 \mathrm{GiB}$, but less than $20 \mathrm{GiB}$.

- Publicly available?: Yes, at https://github.com/diku-dk/ futhark-ppopp19.

- Code/data licenses (if publicly available)?: ISC (noncopyleft free license).

- Archived?: At ACM Digital Library; DOI: 10.1145/3300173.

\subsection{Description}

\subsubsection{How delivered}

The artifact is available (with further documentation and details) from the ACM Digital Library with DOI 10.1145/3300173, and at https://github.com/diku-dk/futhark-ppopp19. It is also available on Docker Hub under the name futhark/ppopp19.

\subsubsection{Hardware dependencies}

A somewhat modern GPU with at least $4 \mathrm{GiB}$ of memory. A significant subset of the experiments can be run with just $2 \mathrm{GiB}$ of memory.

\subsubsection{Software dependencies}

OpenCL to communicate with the GPU, and optionally also CUDA.

\subsubsection{Data sets}

Included in artifact (or downloaded automatically as needed). 


\subsection{Installation}

Using Docker (recommended for x86-64) On a Linux machine with an NVIDIA GPU, the CUDA framework, and NVIDIA's docker runtime $^{1}$, run the following command to enter a Docker instance with the artifact and its various requirements pre-installed:

docker run -it --runtime=nvidia \

--storage-opt size=20G futhark/ppopp19

This may require root permissions, depending on the local system. The artifact also contains a packaged Docker image file, futhark-ppopp19. docker. This image is self-contained, in that it contains pre-downloaded versions of all resources that are otherwise downloaded from the Internet (datasets and benchmark reference implementations), so its contents can be useful for machines that are offline, or if the required resources disappear from the Internet in the future.

Installing Manually Unpack the artifact archive or clone the Git repository, taking care to also include the submodules that contain the Futhark compiler and the FinPar benchmark suite:

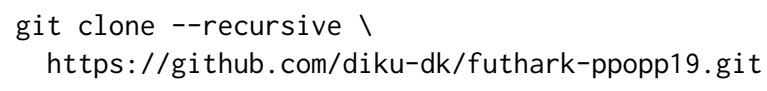

To install the software dependencies on a Debian/Ubuntu system, run the following commands (possibly as root):

apt install binutils build-essential nvidia-cuda-toolkit $\backslash$ sqlite3 libsqlite3-dev libtinfo-dev python-pip git curl \ wget bc libffi-dev libgmp-dev zlib1g-dev texlive $\backslash$ texlive-latex-extra texlive-fonts-recommended । dvipng locales

pip install opentuner matplotlib

curl -sSL https://get.haskellstack.org/ I sh

In case of ambiguities, the Dockerfile also serves as a list of commands necessary to install the required packages.

\subsection{Evaluation and expected result}

- make matmul-runtimes-large.pdf and make matmul-runtimes-small.pdf should construct two graphs similar to the ones making up Figure 2 in the paper. They may differ quantifiably depending on the system, but the curves should have the same rough shape.

- make LocVolCalib-runtimes.pdf should construct a graph similar to the ones in Figure 7.

- make bulk-impact-speedup.pdf should construct a graph similar to the ones in Figure 8.

\footnotetext{
${ }^{1}$ https://github.com/NVIDIA/nvidia-docker\#quickstart
}

We have noticed that some benchmarks (particularly srad and LocVolCalib) may not reliably reach the near-optional thresholds when auto-tuned. If necessary, the maximum time used for autotuning can be increased from the default of thirty minutes in the config.mk file, and e.g. srad rerun with:

$\mathrm{rm}$ results/srad-incremental-tuned.json

make results/srad-incremental-tuned.json

This flaw is a combination of the inherently stochastic nature of autotuning, and a still somewhat brittle attempt on our part to reduce autotuning durations on average.

\subsection{Experiment customization}

Adding a new Futhark implementation of a benchmark simply requires adding a . fut program to the benchmarks directory, with a specially formatted header indicating which workloads to use. Consult the existing programs or the Futhark User's Guide ${ }^{2}$ for more information. Adding a new dataset requires modifying the header for one of the existing . fut files. By convention, the program benchmarks/foo. fut has its datasets in benchmarks/foo-data. After a program benchmarks/foo. fut is added, it can be benchmarked by running

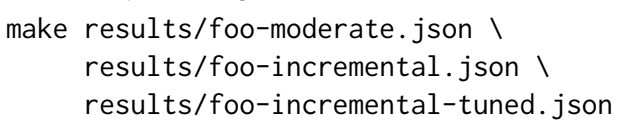

Adding a new reference implementation of a benchmark (e.g. one from Rodinia) is done in an ad-hoc fashion. In particular, Rodinia implementations must be manually modified with instrumentation to perform measurements. The main plotting script (tools/bulk-impact-plot.py) is relatively easy to extend with new benchmarks and datasets (albeit with exactly two datasets per benchmark) by modifying the programs variable.

\subsection{Notes}

While Futhark code tends to be fairly robust in foreign environments, we cannot guarantee the same for the reference benchmark implementations (FinPar and Rodinia) we use. The README. md in the Git repository contains information on how to run only the Futhark implementations, if necessary.

\subsection{Methodology}

Submission, reviewing and badging methodology:

- http://cTuning.org/ae/submission-20180713.html

- http://cTuning.org/ae/reviewing-20180713.html

- https://www.acm.org/publications/policies/artifact-review-badging

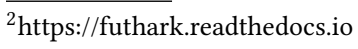

